\title{
Cytogenetic analysis (GTG, CBG and NOR bands) of a wild boar population (Sus scrofa scrofa) with chromosomal polymorphism in the south-east of Spain
}

\author{
JJ Arroyo Nombela ${ }^{1 *}$, C Rodriguez Murcia ${ }^{1}, \mathrm{~T}$ Abaigar ${ }^{2}$, JR Vericad ${ }^{2}$ \\ 1 Laboratorio de Citogenética Animal CSIC c/ Serrano 113, 28006 Madrid; \\ ${ }^{2}$ Estación Experimental de Zonas Aridas CSIC c/ General Segura 1, \\ 04001 Almería, Spain
}

(Received 2 January 1989; accepted 23 September 1989)

\begin{abstract}
Summary - The karyotypes of 12 wild boars $\left(4 \mho^{7}, 8 \%\right)$ from populations in Sierra Nevada and Sierra de Baza (Almería) were analysed. Chromosomal polymorphism giving

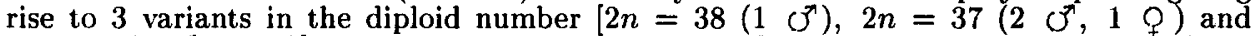
$2 n=36\left(1 \mho^{7}, 7 \bigcirc\right)$ ] was observed. By means of GTC-band analysis, this polymorphism proved to be the consequence of a Robertsonian translocation between members of pairs 15 and 17 of karyotypes with $2 n=38$, thereby creating a long submetacentric chromosome $(15 / 17)$ together with 2 free acrocentric chromosomes, (15 and 17) in individuals with $2 n=37$, and a pair of long submetacentric chromosomes $(15 / 17)$ in $2 n=36$ specimens.

CBG-band analysis revealed 2 types of staining for heterochromatin: a dark staining, characteristic of acrocentric chromosomes and occasionally present in the submetacentric $(15 / 17)$, and a pale staining, characteristic of the rest of the submetacentric chromosomes. The Y-chromosome presented dark heterochromatin over the entire length of its long arm. The nucleolus organizers were observed on pairs 8 and 10 and showed heteromorphism of the size of the silver block and in the distribution in both pairs.
\end{abstract}

wild board / karyotype / chromosomal polymorphism / nucleolus organizer region / heterochromatin

Résumé - Analyse cytogénétique (bandes GTG, CBG et NOR) d'une population de sangliers (Sus scrofa scrofa) et de son polymorphisme dans le Sud-Est de l'Espagne - Les caryotypes de 12 sangliers (4 mâles et 8 femelles) provenant de la Sierra Nevada et Sierra de Baza (Almeria) ont été analysés. Un polymorphisme chromosomique a été observé donnant 3 variantes en ce qui concerne le nombre diploïde de chromosomes: $2 \mathrm{n}=38$ ( 1 mâle); $2 \mathrm{n}=37$ (2 mâles, 1 femelle), $2 \mathrm{n}=36$ ( 1 mâle, 7 femelles $).$ En utilisant les bandes $G T G$ on a montré que le polymorphisme est la conséquence d'une translocation robertsonnienne entre les représentants des paires 15 et 17 des caryotypes ayant $2 \mathrm{n}=38$; créant ainsi un long submétacentrique $(15 / 17)$ avec en même temps 2 acrocentriques isolés, 15 et 17 , chez les individus $2 \mathrm{n}=36$. Une analyse $\dot{a}$ l'aide

* Correspondence and reprints 
des bandes $C B G$ a montré 2 sortes de coloration pour l'hétérochromatine: une coloration sombre caractéristique des chromosomes acrocentrique et parfois présente dans les submétacentriques (15/17), et une coloration claire caractéristique des autres chromosomes submétacentriques. Le chromosome $Y$ présente de l'hétérochromatine sombre sur toute la longueur de ses bras. Les organisateurs nucléolaires ont été observés sur les paires 8 et 10 et ont montré une différence dans la taille des paquets d'argent et dans leur distribution au niveau de 2 paires.

sanglier / caryotype / polymorphisme chromosomique / organisateur nucléolaire / hétérochromatine

\section{INTRODUCTION}

In spite of the abundance and importance of wild boars in the peninsular regions of Spain, little is known about the chromosomal make-up of these animals. This lack of karyological data has motivated our cytogenetic studies of this South-West European population of wild boars. In addition, this research supplements similar studies of wild boar populations in other European regions, such as Germany (McFee et al, 1966; Gropp et al 1969), Yugoslavia (Zivkovic et al, 1971), the Netherlands (Bosma, 1976), Sweden (Gustavsson et al, 1973), France (Mauget et al, 1977; Popescu et al, 1980), Switzerland (Jotterand-Bellomo and Baettig, 1981), Austria (Rittmannsberger, 1971; Mayr et al 1984), and Russia (Tikhonov and Troshina, 1975; Troshina and Tikhonov, 1980).

\section{MATERIAL AND METHODS}

The study comprised 12 specimens (4 $\sigma^{7}, 8 \%$ ) of a Sus scrofa scrofa population, all native to Sierra Nevada and Sierra de Baza (Almería, Spain). The karyotype was obtained from whole blood cultures. Blood samples were taken by venous puncture from anaesthesized males $(n=4)$ and by means of cardiac puncture in the females $(n=8)$. The cultures consisted of $0.4 \mathrm{ml}$ blood in $7.5 \mathrm{ml}$ RPMI-1640 medium supplemented with $15 \%$ fetal bovine serum; $L$-glutamin, antibiotics and phytohaemaglutinin.

The preparations were analysed by the following banding techniques: GTG (Seabright, 1972), CBG (Sumner, 1972) and silver nitrate staining, NORs, (Bloom and Goodpasture, 1976). Using the same methodology, we analysed the chromosomes of 4 hybrids from a male wild boar and a domestic sow.

\section{RESULTS}

Chromosome analysis carried out with conventional Giemsa staining enabled us to verify the existence of numerical variability in the chromosomal sets among the animals analysed. Among the 12 animals analysed, 1 of the males possessed $2 n=38,2$ males and 1 female were $2 n=37$, and 7 females and 1 male were $2 n=36$. This numeric variability was correlated with a morphological variability in 2 chromosomal paires. However, no phenotypical variability was observed.

Application of different banding techniques allowed us to analyse the cytogenetic mechanisms involved in the numerical and structural variability encountered in the 3 variants described above. 


\section{GTG bands}

The metaphases treated with proteolytic enzymes (GTG bands) showed that the karyotype of the male wild boar with $2 n=38$ (Fig 1) consisted of 5 submetacentric chromosomal pairs (1-5), 2 subacrocentric pairs (6-7), 5 metacentric pairs (8-12) and 6 acrocentric pairs (13-18), plus the 2 gonosomes (metacentric X-chromosome, of similar length to the autosomal pair 9, and a small metacentric $Y$ ). This karyotype is identical (in number of chromosomes, morphology and banding patterns) to the karyotype of the domestic pig (Halgeltorn and Gustavsson, 1973; Lin et al, 1980).

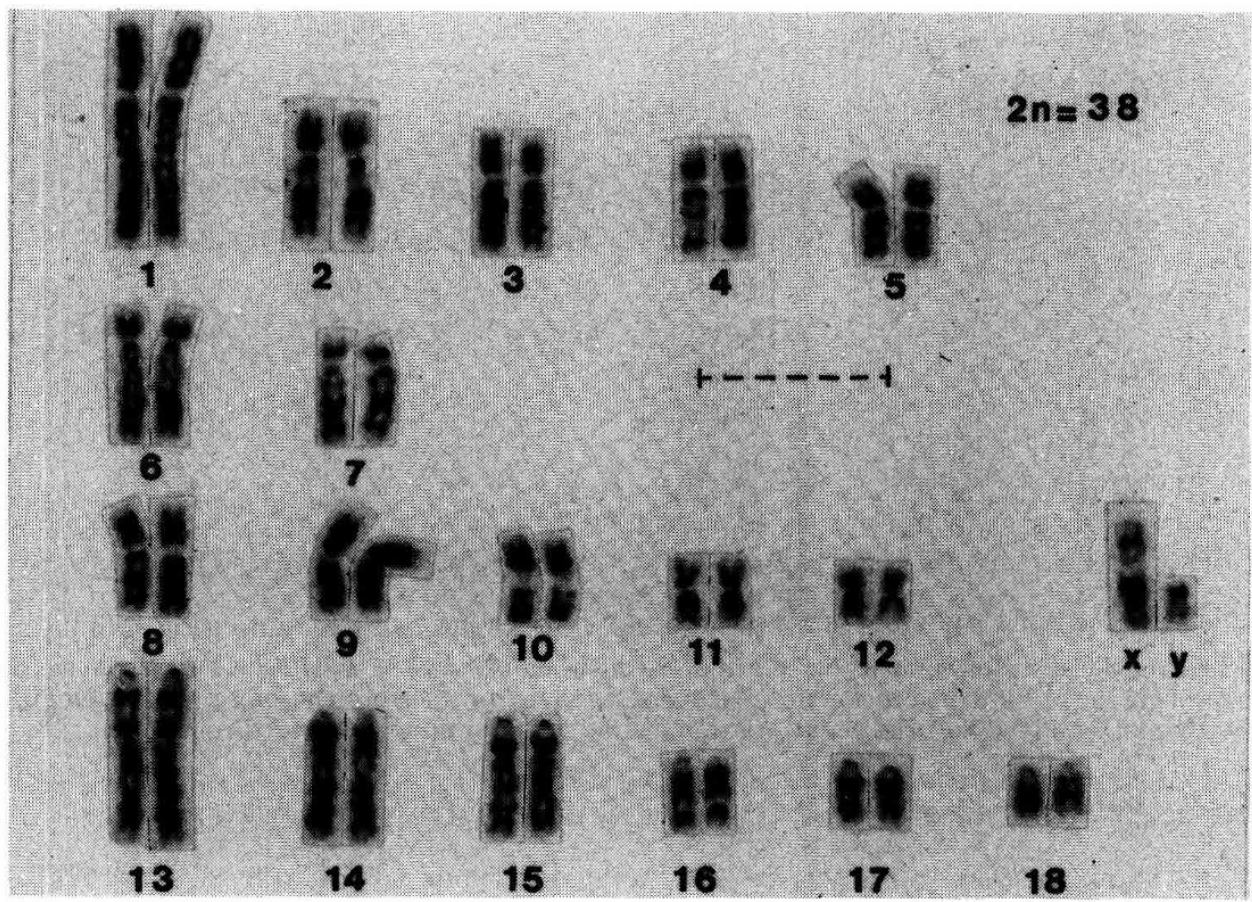

Fig 1. GTG-banded karyotype of male wild boar $(2 n=38)$. Bar in all Figures represents $10 \mu \mathrm{m}$.

The metaphases with $2 n=37$ (Fig 2) presented structural heteromorphism in pairs 15 and 17, resulting in 2 unpaired acrocentric chromosomes (see arrows in Fig 2) and a long submetacentric chromosome (15/17) with a length intermediate between pairs 1 and 2 . The $p$ and $q$ arms of this submecentric chromosome had banding patterns and relative length identical to the 2 acrocentric chromosomes 15 and 17. The other pairs, including the gonosomes, were identical to those of the individuals, with $2 n=38$. A Robertsonian translocation (centric fusion) between 1 chromosome, each of pairs 15 and 17, would account for the heteromorphism found in the $2 n=37$ specimens, and hence, explain the numerical variation in the diploid chromosome number. 
Finally, the metaphases with $2 n=36$ (Fig 3 ) lacked the unpaired acrocentrics ( 15 and 17) characteristic of $2 n=37$, but contained a pair of long submetacentric $(15 / 17)$ chromosomes.

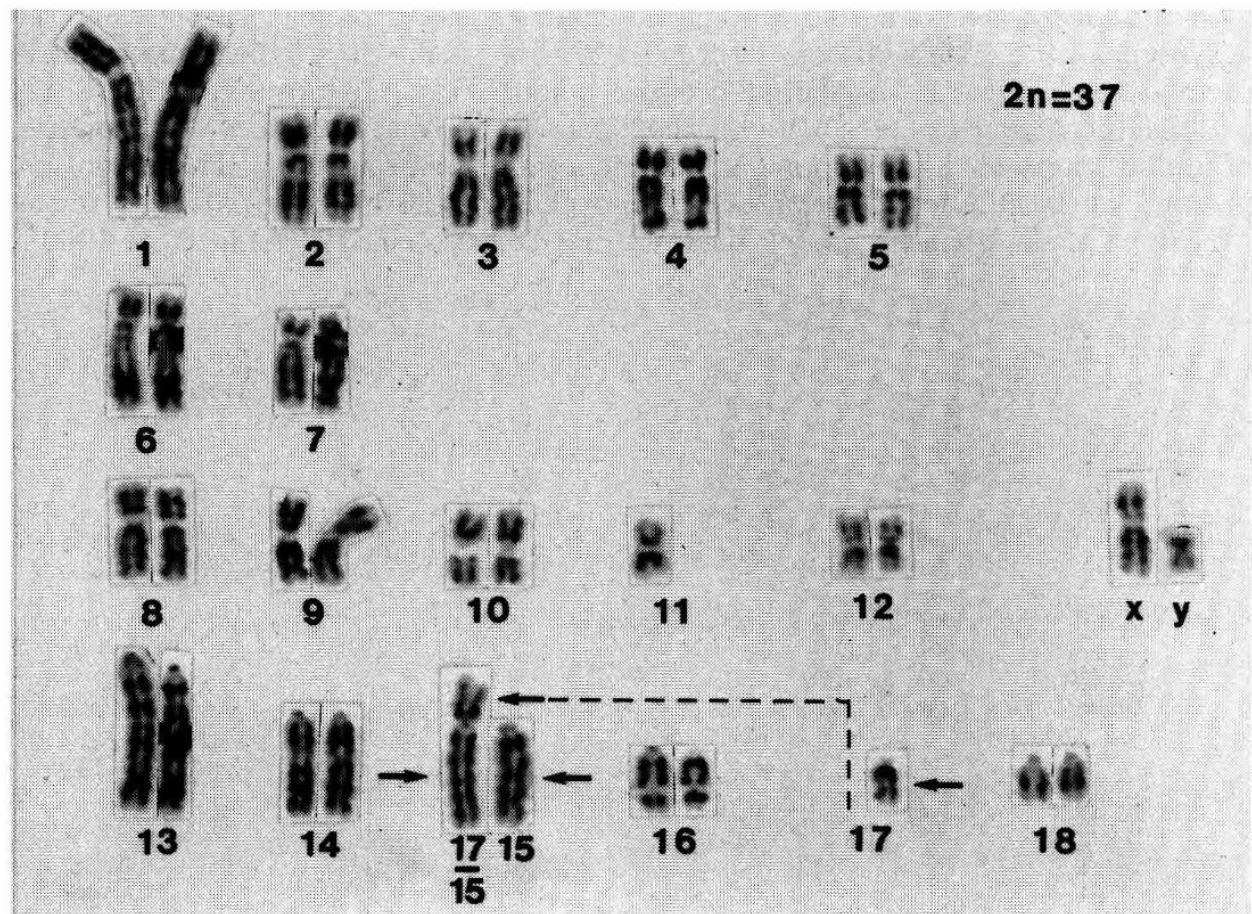

Fig 2. GTG-banded karyotype of a male wild boar $(2 n=37)$, with 1 submetacentric, 1 accrocentric 15, and 1 acrocentric 17 chromosome (arrows). Note the banding homologies between 15 and 17 and the $p$ and $q$ arms of the $15 / 17$ submetacentric chromosome.

\section{CBG bands}

CBG-band analysis (Summer, 1972), revealed 2 types of centromeric heterochromatin in the karyotypes: a darker staining which proved to be alkali resistant and very constant and which was found in all acrocentric chromosomes, and a pale, alkali-labile staining associated with the subtelocentric and meta-submetacentric chromosomes. The heterochromatin blocks of the acrocentric chromosomes varied in size, even for chromosomes of the same pair, but were observed for all chromosomes of this morphological class. In contrast, the heterochromatin of non-acrocentric pairs was pale (at times not even detectable) and showed a graded staining pattern according to the pairs, but was particularly constant in pair 1 . The (15/17) chromosome was exceptional in having a double block of dark-staining centromeric similar to that of the unpaired acrocentric chromosomes 15 and 17 (Figs 4 and 5). Frequently, a kind of intercalary heterochromatin was detected in the long arm of pair 10 in the proximity of the secondary constriction, which correspond to the nucleolus organizer of this pair. The long arm of the Y-chromosome was totally 


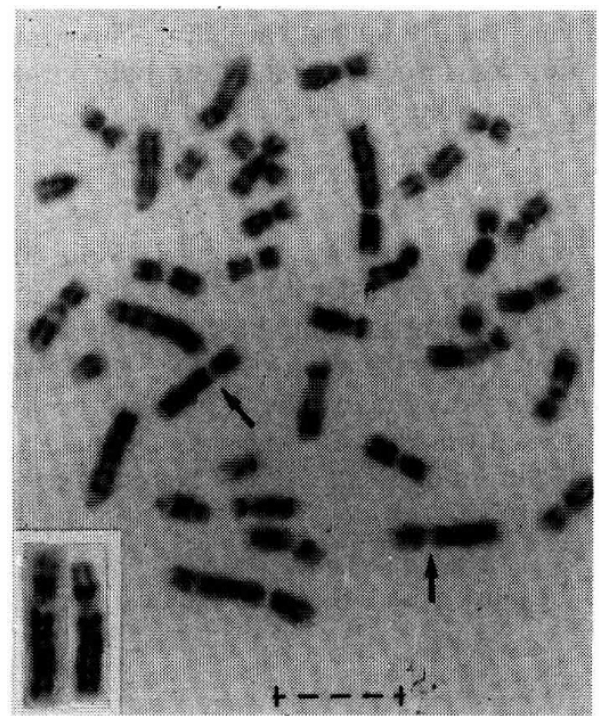

Fig 3. GTG metaphase of a female wild boar $(2 n=36)$ with 2 submetacentric (15/17) chromosomes (arrows). Higher magnification of this pair is shown in the lower left-hand corner.

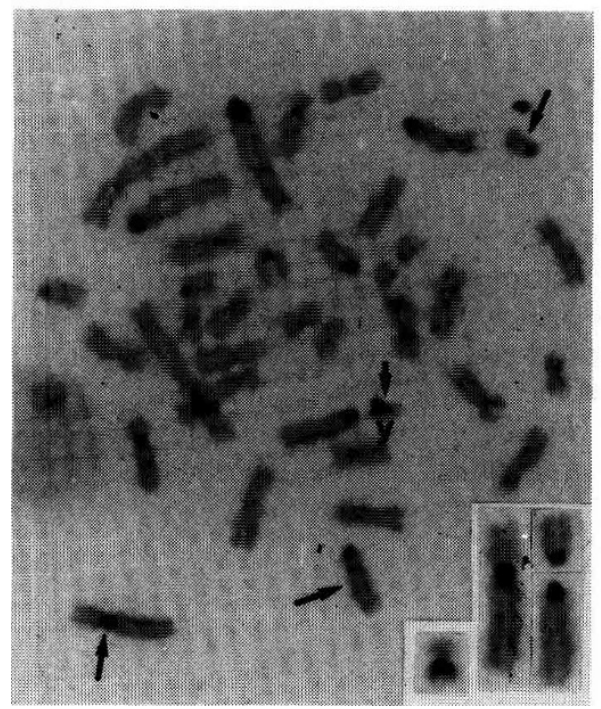

Fig 5. CBG-banded metaphase of male $(2 n=37)$ with 1 submetacentric $(15 / 17)$ chromosome showing double block dark staining centromeric heterochromatin and number 15 and 17 chromosomes with dark staining centromeric heterochromatin. Higher magnification of these chromosomes and the Y-chromosome is shown in the lower right-hand corner.

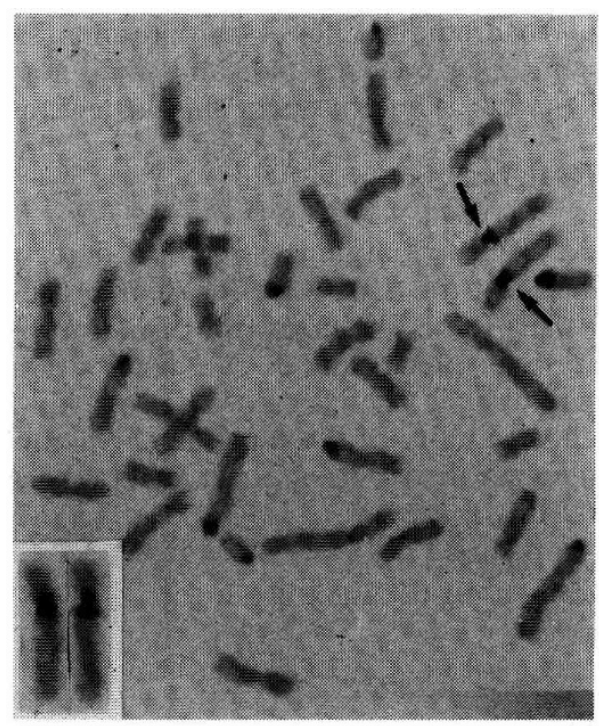

Fig 4. CBG-banded metaphase of a female $(2 n=36)$. Note the double block of dark staining centromeric heterochromatin in both submetacentric (15/17) chromosomes (arrows). Higher magnification of this pair is shown in the lower left-hand corner.

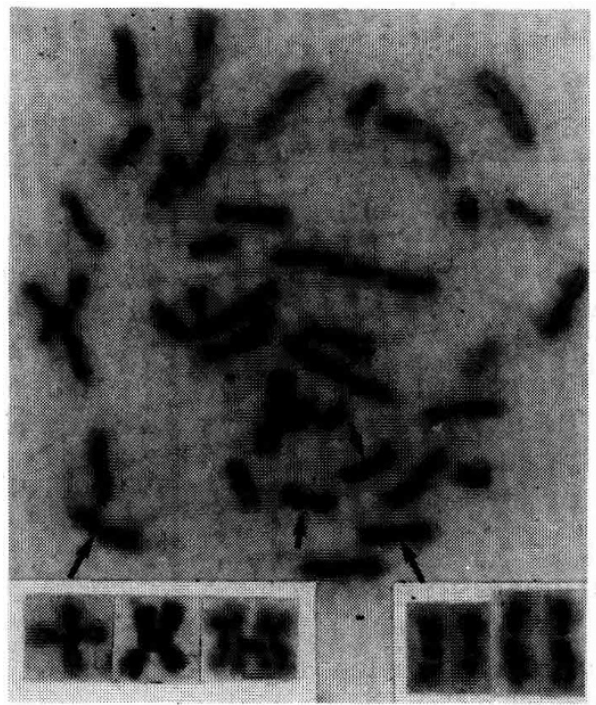

Fig 6. Silver stained metaphase. Note the NOR expression on 4 chromosomes (pairs 8 and 10) showing different size blocks on both homologous chromosomes number 10 (short arrows). Bottom: higher magnification of these pairs and 3 tetraradial structures by association with chromosomes number 10. 
heterochromatic. The X-chromosome, however, usually lacked the heterochromatin staining, or was very pale and could not be differentiated from the heterochromatin of the autosomal pairs of similar length and morphology (pairs 8 and 9).

\section{NOR bands}

The nucleolus organizer regions, detected by means of silver staining (Bloom and Goodpasture, 1976) are located in the paracentrometric region of the short arm of pair 8 and the long arm of pair 10 (Fig 6). Polymorphism, both in the size of the silver blocks and in the distribution within the pair, was observed. Block size was larger in the chromosomes of pair 10, and even within this pair there existed size variability of the block in the homologous chromosomes. Analysis of nucleolus organizer distribution on 347 metaphases of 4 animals $\left(20^{7}, 2\right.$ ) is summarized in Table I.

Table I. Distribution of silver stained NORs on pairs 8 and 10 chromosomes in 4 wild boars.

\begin{tabular}{|c|c|c|c|c|c|c|c|c|}
\hline \multirow[t]{2}{*}{$\begin{array}{l}\text { Animal } \\
\text { number }\end{array}$} & \multirow[t]{2}{*}{ Sex } & \multirow[t]{2}{*}{$2 n$} & \multicolumn{4}{|c|}{$\begin{array}{c}\text { Stained metaphases } \\
\text { Ag-stained chromosomes }\end{array}$} & \multirow[t]{2}{*}{$\begin{array}{c}\text { Unstained } \\
\text { metaphases }\end{array}$} & \multirow{2}{*}{$\begin{array}{l}\text { Total } \\
\text { number of } \\
\text { analysed } \\
\text { metaphases }\end{array}$} \\
\hline & & & 10 & $10-10$ & $10-10,8$ & $10-10,8-8$ & & \\
\hline 1 & 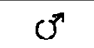 & 38 & 5 & 145 & 3 & 2 & 5 & 160 \\
\hline 2 & $\circlearrowleft$ & 37 & 5 & 37 & 2 & 0 & 16 & 60 \\
\hline 3 & O & 36 & 6 & 34 & 3 & 0 & 20 & 63 \\
\hline \multirow[t]{3}{*}{4} & ? & 36 & 11 & 34 & 2 & 2 & 15 & 64 \\
\hline & Total & & 27 & 250 & 10 & 4 & 56 & 347 \\
\hline & $\%$ & & 7.75 & 72.06 & 2.9 & 1.15 & 16.14 & 100 \\
\hline
\end{tabular}

Table I shows that $84 \%$ of the metaphases contained some active nucleolus organizer regions. Of these, $80 \%$ were from metaphases with at least 1 active chromosome in pair 10 , while $72 \%$ of metaphases showed staining in both chromosome of pair 10 . Only $4 \%$ presented staining in a chromosome of pair 8 , and only $1.15 \%$ of the metaphases showed 4 active chromosome.

In addition, chromosomal associations were observed in pair 10, giving rise to tetraradial structures (Fig 6b), a phenomenon which was not recorded for pair 8 , or between pairs 8 and 10 .

\section{DISCUSSION}

This study provides evidence that Spanish wild boars present cytogenetic characteristics which show a high degree of similarity to those of other European populations (McFee et al, 1966; Gropp et al, 1969; Zivkovic et al, 1971; Gustavsson et al, 1973; Bosma, 1976, Mauget et al, 1977; Popescu et al, 1980; Jotterand-Bellomo and Baettig, 1981; Mayr et al, 1984). 
In fact, the wild boar and domestic pig karyotypes are very similar. In specimens with $2 n=38$, it proved to be impossible cytogenetically to differentiate between subspecies, as they showed identical features in the number of chromosomes, pair morphology and even banding patterns for both gonosomes and autosomes. In addition, the Spanish wild boar population presents a numerical polymorphism with 3 variants in their chromosomal sets $(2 n=36,37$ and 38$)$ as a consequence of a translocation involving pairs 15 and 17. A similar polymorphism has also been observed in German wild boar populations studied by McFee et al (1986), in the Dutch specimens (Bosma, 1976), as well as in the Austrian population reported by Mayr et al, (1984). However, the German specimens analysed by Gropp et al (1969) as well as the French studies (Mauget et al, 1977; Popescu et al, 1980) and the Swiss population (Jotterand-Bellomo and Beattig, 1981), did not present this polymorphism and are homogenous populations with $2 n=36$. Finally, Zivkovic et al (1971) discovered $2 n=38$ sets in certain specimens native to different regions in Yugoslavia, and Popescu et al, (1980) in animals from the island of Corsica. These studies confirm the variability in the wild boar karyotype which contrasts with the constancy found for domestic pig karyotype, independent of origin (Hansen, 1972; Gustavsson et al, 1972; Berger, 1972; Hageltorn and Gustavsson, 1973; HansenMelander and Melander, 1974; Ford et al, 1980; Lin et al, 1980; Ronne et al, 1987).

In the light of the analogies between the wild boar and domestic pig karyotypes differentiated only by a Robertsonian translocation, one can only speculate about the ancestral karyotype of these species. Are the 15 and 17 acrocentric chromosomes, or the $15 / 17$ submetacentric chromosomes the ancestral morphology? The involvement of chromosome 17 in 2 Robertsonian translocations [(15/17) in the European wild boar and (16/17) in the Asian species (Tikhonov and Troshina, 1975)] suggests fusion as the most likely process during evolution, and hence, the ancestral karyotype most likely contained the acrocentric chromosomes. On the other hand, the centrometric heterochromatin of the submetacentric pair 15/17, unlike the other meta-submetacentric chromosomes, showed dark staining similar to acrocentric chromosomes. This exception, in turn, allows for speculation about the ancestral character of the acrocentric chromosomes. It is legitimate to assume the dark heterochromatin to be ancestral heterochromatin and the pale heterochromatin to be a derivative of the former.

This assumption is indirectly supported by evidence provided by Arroyo Nombela and Rodriguez Murcia (1977), who reported a spontaneous double Robertsonian translocation which appeared in a colony of NMRI mice, showing that the resulting biarmed chromosomes $(2,3)$ and $(\mathrm{X}, 3)$ had dark-stained double heterochromatin block and even retained the relative lengths of the acrocentric chromosome blocks from which they had originated. Hence, the translocation does not alter the nature of the heterochromatin. Moreover, its differentiation would occur at a later stage as a gradual adaptive process of the rearrangement in the population extending over a long period of time. Both kinds of heterochromatin are generalized in the karyotype of mammals with acrocentric and metacentric chromosomes. Furthermore, we also found this occurring in Gazella dama mhorr (unpublished data) with graded staining in the different meta-submetacentric data pairs. This grading could be taken as an indicator of the rearrangement chronology. 
Furthermore, we suggest that the ancestral karyotype with $2 n=38$ gave rise to European wild boar species by means of a 15/17 Robertsonian translocation, and to the Asian wild boar variety by means of another 16/17 translocation.

The Robertsonian translocations differentiating the domestic pig and the wild boar karyotypes do not inhibit hybridization of the subspecies. We have examined a litter of 4 young wild boars which resulted from the breeding of a male wild boar and a domestic sow. The descendants showed sets of $2 n=38$ and 37. In this context, the work of Troshina and Tikhonov (1980) on Landrace pig and and European wild boar (15/17), as well as Asian wild boar (16/17) hybrids, is highly conclusive, demonstrating that neither of the 2 translocations constitute an isolating reproductive barrier, and that viable and fertile hybrids were obtained from crosses of these subspecies, resulting in $2 n=36(34+15 / 17+16 / 17)$. In this light, it seems legitimate to consider Sus scrofa domestica, Sus scrofa scrofa and Sus scrofa nigripens as 3 karyotypic races.

It is not clear whether the present numerical polymorphism observed is due to a recent translocation or to interbreeding of domestic pig and wild boar. The size and general presence of the silver blocks on the pair 10 in all analysed individuals cause us to think that this pair must be considered as the main site of r-RNA production.

No phenotypic variability was observed in any of the analysed specimens, which have the appearance of true typical wild boars. Usually Robertsonian polymorphisms have no phenotypic expression.

\section{ACKNOWLEDGMENTS}

We are grateful to $\mathrm{Mr} \mathrm{A}$ Rueda and $\mathrm{Mr} \mathrm{J}$ Vallejo for generous assistance in the field.

\section{REFERENCES}

Arroyo Nombela JJ, Rodriguez Murcia C (1977) Spontaneous double Robertsonian translocation $\mathrm{Rb}$ (2.3) and $\mathrm{Rb}$ (X.3) in the mouse. Cytogenet Cell Genet 19, 227-230

Berger R (1972) Étude du caryotype du porc avec une nouvelle technique. Exp Cell Res 75, 298-300

Bloom SE, Goodpasture C (1976) An improved technique for selective silver staining of nucleolar organizer regions in human chromosomes. Hum Genet 34, 199-206

Bosma AA (1976) Chromosomal polymorphism and G-banding patterns in the wild boars (Sus scrofa L) from the Netherlands. Genética 46, 391-399

Ford CE, Pollock DL, Gustavsson I (1980) Proc 1st Int Conf for the Standardization of Banded Karyotype of Domestic Animals. Hereditas 91, 142-162

Gropp A, Giers D, Tettenborn U (1969) Das Chromosomenkomplement des Wildschweins (Sus scrofa). Experientia 25, 778

Gustavsson I, Hageltorn J, Johanson C, Zech L (1972) Identification of the pig chromosomes by the quinacrine mustard fluorescent technique. Exp Cell Res 70, 471-474 
Gustavsson I, Hagelthorn M, Zech L, Reiland S (1973) Identification of the chromosomes in centric fusion, fission polymorphic system of the pig (Sus scrofa L) Hereditas 75, 153-155

Hagelthorn M, Gustavsson I (1973) Giemsa staining patterns for identification of the pig mitotic chromosomes. Hereditas 75, 144-146

Hansen KM (1972) The karyotype of the pig (Sus scrofa domestica) identified by quinacrine mustard staining and fluorescent microscopy. Cytogenetics 11, 286-294 Hansen-Melander E, Melander Y (1974) The karyotype of the pig. Hereditas 77, 149-158

Jotterand-Bellomo M, Baettig M (1981) Étude cytogénétique de deux sangliers (Sus scrofa) de couleur claire capturés aux environs de Genève (Suisse). Rev Suisse Zool 88, 787-795

Lin CC, Biederman BM, Jamro HK, Hawthorne AB, Church RB (1980) Porcine (Sus scrofa domestica) chromosome identification and suggested nomenclature. Can $J$ Genet Cytol 22, 103-116

Mauget R, Castet MC, Maraud R, Canivenc R (1977) Étude dynamique et caryotypique d'une population de sangliers à robe claire. CR Seanc Soc Biol 171, 592-596

Mayr B, Schweizer D, Geber G (1984) NOR activity heterochromatin differentiation and the Robertsonian polymorphism in Sus scrofa L. J Hered 75, 79-80

McFee AF, Banner MW, Rary JM (1966) Variation in chromosome number among European wild pigs. Cytogenetics 5, 75-81

Popescu CP, Quere JP, Francesci P (1980) Observations chromosomiques chez le sanglier français (Sus scrofa scrofa). Ann Génét Sél Anim 12, 395-400

Rittmannsberger C (1971) Chromosomenuntersuchungen bei Wild und Hausschweinen. Ann Génét Sél Anim 3, 105-107

Ronne M, Stefanova V, Di Berardino D, Strandby Poulsen B (1987) The R-banded karyotype of the domestic pig. Sus scrofa domestica L. Hereditas 106, 219-231

Seabright M (1972) The use of proteolytic enzymes for mapping of structural rearrangement in the chromosomes of man. Chromosoma 36, 204-210

Sumner AT (1972) A simple technique for demonstrating centrometric heterochromatin. Exp Cel Res 75, 304-306

Tikhonov VN, Troshina AI (1975) Chromosomes translocations in the karyotypes of wild boars. Sus scrofa L of the European and the Asian Areas of USSR. Theor Appl Genet 45, 304-308

Troshina AI, Tikhonov VN (1980) The reproductive features of Landrace $\times$ wild boar hybrid with two chromosomal translocation $\operatorname{Tr} 1$ (16/17) $\operatorname{Tr} 2$ (15/17). 4th Eur Colloq Citogenet Domest Anim, pp 250-261

Zivkovic S, Jovanovic V, Isakovic I, Milosevic M, (1971) Chromosome complement of the European wild pig. (Sus scrofa L) Experientia 27, 224-226 